\title{
Diagnostics of Coronal Magnetic Field in Terms of Radio Burst and Fine Structures
}

\author{
M. Wang ${ }^{1} \dagger$, C. $\mathrm{Xu}^{1}$, R. X. Xie ${ }^{1}$, and Y. H. Yan ${ }^{2}$ \\ ${ }^{1}$ National Astronomical Observatories/Yunnan Observatory, Chinese Academy of Sciences, \\ Kunming 650011, China (email: wmynao@163.net) \\ ${ }^{2}$ National Astronomical Observatories, Chinese Academy of Sciences, Beijing 100012, China
}

\begin{abstract}
A complex solar radio burst was observed on 19 October 2001 with the spectrometers of NAOC (National Astronomical Observatory of China) and Nobeyama Radioheliograph $(\mathrm{NoRH})$. Basing on the analysis of brightness temperature spectra of radio sources and various fine spectral structures, we get a diagnosis of magnetic field of radio active region.
\end{abstract}

Keywords. Sun: coronal mass ejections (CMEs), magnetic fields, radio radiation

\section{Introduction}

Radio spectral observation is effective for studying the acceleration process, while the image observation is effective for studying the magnetic field configuration. Basing on a analysis of a solar radio burst observed by the spectrometer $(0.65-7.60 \mathrm{GHz})$ of NAOC and NoRH, we give a diagnosis of magnetic fields in the radio active regions.

\section{Observations and data analysis}

\subsection{NoRH observation and magnetic field diagnosis}

In the radio image of $\mathrm{NoRH}$, we find three sources, one is a pre-existing source named PS and the others are newly emerging sources named NES1 and NES2, respectively. From the polarization degrees of Sources NES1 and PS, we can calculate the range of $\theta$ (the angle between the line of sight and the magnetic fields), under the assumption that the emissions of these two sources are due to non-thermal gyro-synchrotron radiation. According to the Equation (38) of Dulk (1985), for the harmonic number $10 \leqslant s \leqslant 50$ and $\delta=3.5$ (for Source NES1) and 5.3 (for Source PS), $\theta$ should be between $83-158^{0}$ for Source NES1 and between $30-66^{0}$ for Source PS. We refer that the peak frequency is $9400 \mathrm{MHz}$ for both Source NES1 and PS according to the data from SGD. According to the Equation (36), (37), and (39) of Dulk (1985), for the reasonable values of NL (about $\left.10^{18-19} \mathrm{~cm}^{-2}\right)$, we get that the values of magnetic field strength are 80-160 and 300-400 Gauss for Source NES1 and PS, respectively.

\subsection{Spectral observation and magnetic field diagnosis}

The highly polarized type III bursts in the frequency range of $2.6-3.8 \mathrm{GHz}$ are due to fundamental plasma emissions. Using the method of Wang et al. (2003), we get the corresponding magnetic field should be about 120 Gauss.

Zebra patterns are due to upper hybrid waves or whistler waves. The frequency space between the Zebra patterns gives the diagnosis to the magnetic field of the source, because the frequency space is roughly equal to the local gyrofrequency. From the observed frequency space of about 30-40 MHz, we get the magnetic field is about 15 Gauss.

$\dagger$ Present address: PO Box 650011, Kunming, Yunnan, China. 


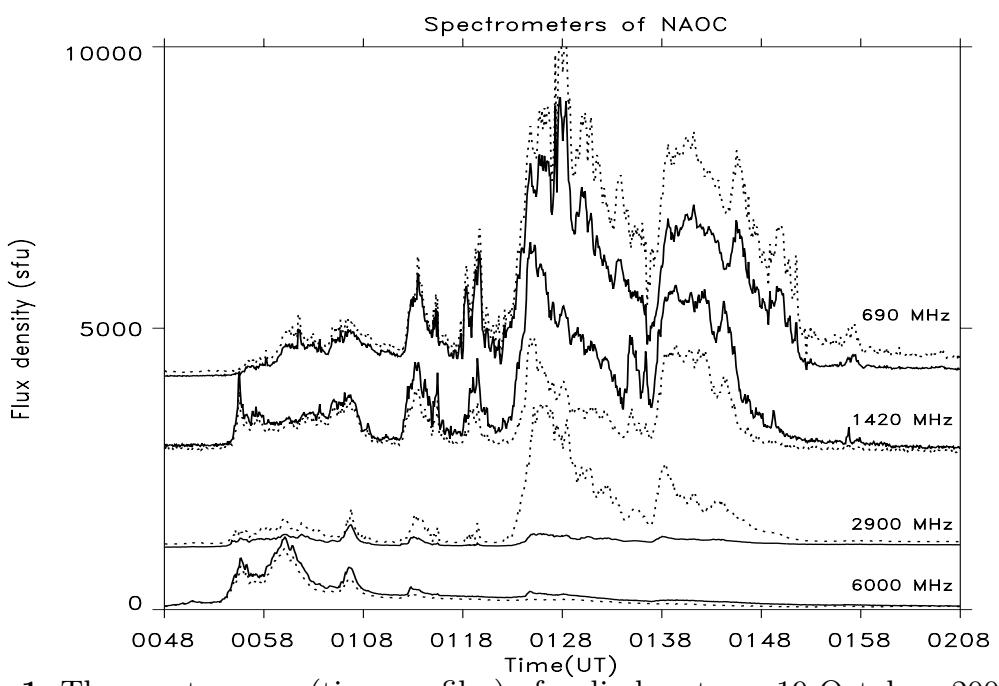

Figure 1. The spectrogram (time profiles) of radio bursts on 19 October, 2001 at selected frequencies, the dashed lines represent the right polarization, the solid lines left polarization.

We found some lower frequency fibers with normal frequency drifting rate of about 25 $\mathrm{MHz} / \mathrm{s}$ and some higher frequency fibers with normal frequency drifting rate of about 90 $\mathrm{MHz} / \mathrm{s}$. The drift rates of fibers are relates to the local Alfvén speed (Treumann, Güdel, and Benz, 1990). For 0.65-0.8 GHz and 2.6-3.8 GHz, we can take density scale height $\lambda_{n}$ be about $10^{10}$ and $0.5 \times 10^{10} \mathrm{~cm}$, respectively. We take frequency $f_{1}=770 \mathrm{MHz}$ and $f_{2}=3000 \mathrm{MHz}$, electron density $n_{1}=1.8 \times 10^{9} \mathrm{~cm}^{-3}$, and $n_{2}=2.7 \times 10^{10} \mathrm{~cm}^{-3}$ (assuming harmonic emission), we get the Alfvén speeds $v_{A 1}=1400 \mathrm{~km} / \mathrm{s}$ and $v_{A 2}=3500 \mathrm{~km} / \mathrm{s}$, that means the local magnetic fields are 210 and 130 Gauss, respectively.

The observed 6-minute and 1-minute pulsations should be attributed to the standing Alfvén wave in the coronal loops driven by the photospheric velocity fields which modulate the radio emission and the kink fast magneto-acoustic mode, respectively. For 1-minute pulsations, according to Aschwanden et al. (1999), for the observed period about $60 \mathrm{~s}$, assuming second harmonic emission, $\mathrm{n}=2.8 \times 10^{10} \mathrm{~cm}^{-3}$ and $\mathrm{L}=0.94 \times 10^{10}$ $\mathrm{cm}$, we get $\mathrm{B}=170 \mathrm{G}$. For 6 -minute pulsations, $\mathrm{L}=5.0 \times 10^{10} \mathrm{~cm}$, according to Strauss, Kaufmann, and Opher (1980), we get the Alfvén speed $\mathrm{V}_{A}=2550 \mathrm{~km} / \mathrm{s}$. At the height of $3 \mathrm{GHz}$ emission, the electron density $\mathrm{n}=1.3 \times 10^{9} \mathrm{~cm}^{-3}$, so the magnetic field $\mathrm{B}=50$ gauss; for the height of $17 \mathrm{GHz}$ emission, $\mathrm{n}=1.0 \times 10^{11} \mathrm{~cm}^{-3}$, we get $\mathrm{B}=380$ Gauss.

\section{Acknowledgements}

This work is supported by the Ministry of Science and Technology of China (No. G2000078403) and the national NSF of China (Nos. 19833050,10333030, and 10473020).

\section{References}

Aschwanden, M. J., Fletcher, L., Schrijver, C. J., \& Alexander, D. 1999, ApJ 520, 880

Dulk, G. A. 1985, ARAA 23, 169

Strauss, F. M., Kaufmann, P., \& Opher, R. 1980, Solar Phys. 67, 83

Treumann, R. A., Güdel, M., \& Benz, A. O. 1990, AA 236, 242

Wang M., Duan, C. C., Xie R. X., \& Yan, Y. H. 2003, Solar Phys. 212, 401 